\title{
Anterior maxillary wall and lacrimal duct relationship - CT analysis for prelacrimal access to the maxillary sinus*
}

\author{
Daniel Simmen', Narendrakumar Veerasigamani', Hans Rudolf Briner', \\ Nick Jones², Bernhard Schuknecht ${ }^{3}$ \\ Center for Otorhinolaryngology, Head and Neck Surgery, Hirslanden Clinic, Zurich, Switzerland \\ 2 Department of Otorhinolaryngology, Head and Neck Surgery, University Hospital, Queens Medical Centre, Nottingham, \\ United Kingdom \\ ${ }^{3}$ Medical Radiological Institute MRI, Zurich, Switzerland
}

Rhinology 55: 170-174, 2017

https://doi.org/10.4193/Rhino16.318

*Received for publication:

November 4, 2016

Accepted: February 8, 2017

\begin{abstract}
Background: The distance between the anterior wall of the maxillary sinus and the nasolacrimal duct shows a large individual variation.
\end{abstract}

Methodology: To evaluate the feasibility of accessing the maxillary sinus through a prelacrimal window access (PLWA), a series of 100 paranasal CT scans from adult patients was analysed retrospectively. The distance between the anterior maxillary wall and the anterior border of the lacrimal duct (= prelacrimal window) were measured in 200 sides.

Results: A distance of more the $7 \mathrm{~mm}$ was found in $12.5 \%$ maxillary sinuses and would enable straight forward PLWA. A distance between $<7 \mathrm{~mm}$ and $>3 \mathrm{~mm}$ was present in $56.5 \%$ of sinuses, and would make surgical access more demanding. In $31.5 \%$ of maxillary sinuses the distance was $\leq 3 \mathrm{~mm}$ and in these patients this approach would be difficult without transecting the nasolacrimal duct.

Conclusion: Only in $12.5 \%$ of sinuses a prelacrimal endoscopic access is readily feasible, while in $56.5 \%$ temporary tear sac dislocation is required and in $31.5 \%$ lacrimal sac dislocation is always needed along with a significant amount of bone removal to enable PLWA.

Key words: maxillary sinus, medial maxillectomy, modified medial maxillectomy, prelacrimal window, alveolar recess, lacrimal recess

\section{Introduction}

Surgical access to pathology within the maxillary sinus may be difficult particularly if the lesion is based at the anterior wall or floor ${ }^{(1)}$. The medial and posterior walls of the maxillary sinus are readily accessible after a type III sinusotomy ${ }^{(2)}$. The medial wall can be resected endoscopically by doing a medial maxillectomy. Lesions along the anterior wall and floor are much more difficult to expose. It may be possible to access these sites after a type III sinusotomy by using a $70^{\circ}$ endoscope, or after an endoscopic medial maxillectomy. Some endoscopic sinus surgeons also do an approach via the canine fossa at the same time in order to help visualize the maxillary sinus ${ }^{(3)}$. Lateral rhinotomy or midfacial degloving also provides good access and visibility to the maxillary sinus and lateral nasal wall ${ }^{(4)}$. The morbidity in both approaches is greater than with an endoscopic modified medial maxillectomy ${ }^{(5)}$.

Recently, Zhou et al. ${ }^{(6)}$ described the prelacrimal window approach (PLWA) by keeping the lacrimal system intact and preserving the inferior turbinate and at the same time obtaining access to the alveolar recess, the prelacrimal recess and anterior wall of the maxillary sinus. Using this access it became possible to preserve the sinus by skeletonizing the lacrimal system before en- 
tering anterolateral to it. Following this approach, it is possible to remove the entire remains of the medial wall in order to allow access to the anterior part of the maxillary sinus. The extent of removal of bone can be tailored to the pathology ${ }^{(2)}$.

In this study we analysed the variations in anatomy between the anterior maxillary wall and the lacrimal duct system. The position of the lacrimal duct in relation to the anterior maxillary wall determines how readily a prelacrimal window can be made. This is important information for the surgeon prior to planning a modified medial maxillectomy approach to the maxillary sinus.

\section{Materials and methods}

\section{Study design}

Retrospective analysis of a consecutive series of 100 paranasal sinus CT examinations obtained in adults ( $>18$ years) was done using a protocol on a 128 Multi-Detector CT (Siemens Sensation AS) in patients with chronic rhinosinusitis following topical steroid treatment for a minimum of 2 weeks. Patient's informed consent was obtained for anonymized anatomic assessment of their CT data. An axial low dose CT data set was acquired that covered the paranasal sinuses from the maxillary alveolar process to the roof of the frontal sinus resulting in a dose $<0.5 \mathrm{mSV}$ (180eff. mAs). The raw data was reconstructed at $0.6 / 0.4 \mathrm{~mm}$ slice thickness/ increment). Multiplanar reconstructions (MPR) were displaced as $0.75 \mathrm{~mm}$ slices in the coronal, sagittal and axial planes.

To assess the available space for surgical access to the maxil- lary sinus in the lateral nasal wall anterior to the lacrimal duct the distance between the anterior wall of the maxillary sinus and the anterior and posterior border of the lacrimal duct was measured using the Picture Archiving and Communication Systems (PACS) workstation for both the right and left side in 100 patients (200 sides).

The measurement was performed by identification of the anterior insertion of the inferior turbinate into the frontal process of the maxilla (coronal plane) (Figure 1) and in the corresponding axial plane by positioning a tangential line through the posterior surface of the anterior wall of the maxillary sinus (line 1) and a parallel line through the anterior (line 2) and posterior wall (line 3 ) of the lacrimal duct (Figure 2). The distances between line 1 and 2 (distance 1) and line 1 and 3 (distance 2) were measured and statistically assessed.

\section{Statistical analysis}

Statistical analysis was performed using Statistical Package for Social Science (SPSS) (v:17). The data were not normally distributed and therefore parametric/ non - parametric tests were performed. Independent t test / Mann Whitney test were used to compare continuous variables between two groups. A Pearson correlation coefficient/ Kendall's tab analysis was used to examine the relationship of two related variables. A chi-squared test was used for comparison between two attributes. A two-sided $p$ value $<0.05$ was considered statistically significant.

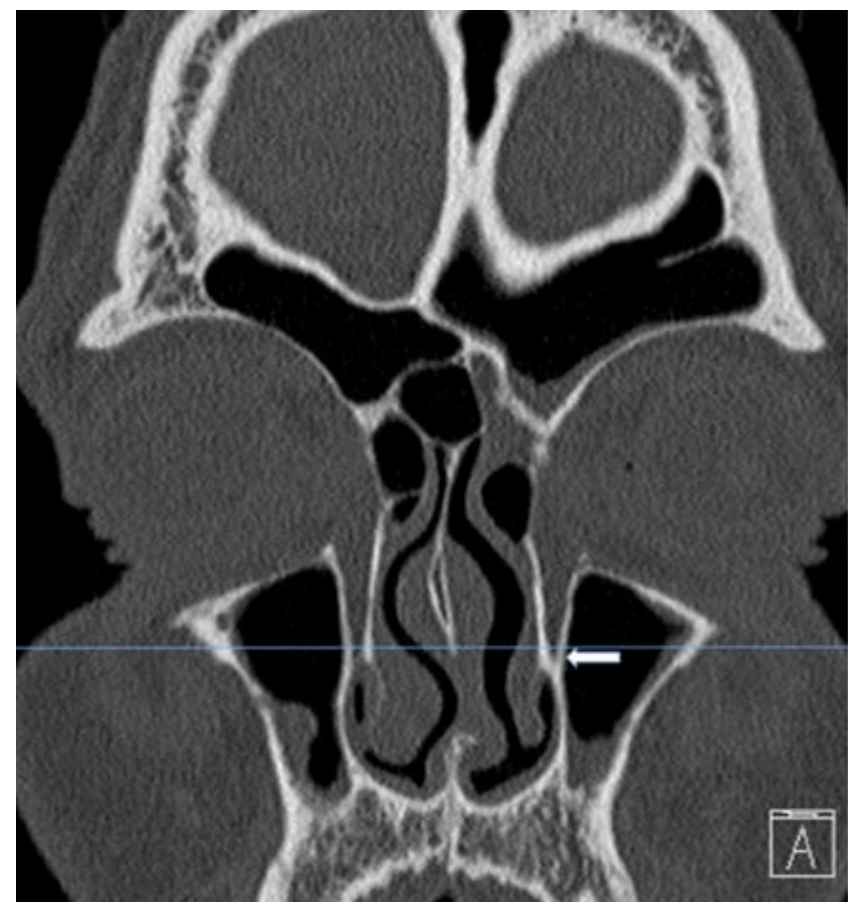

Figure 1. Coronal CT slice depicts the junction of the "shoulder" of the inferior turbinate with the frontal process of the maxilla (White arrow).

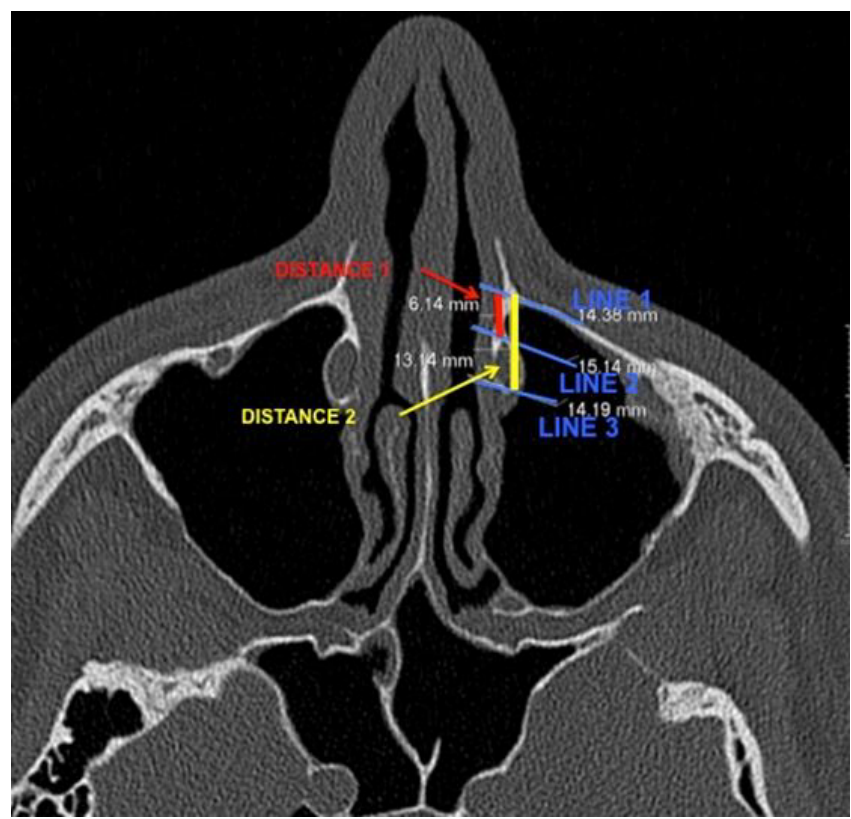

Figure 2. Corresponding axial CT slice left side (Red line - distance 1, yellow line - distance 2). 


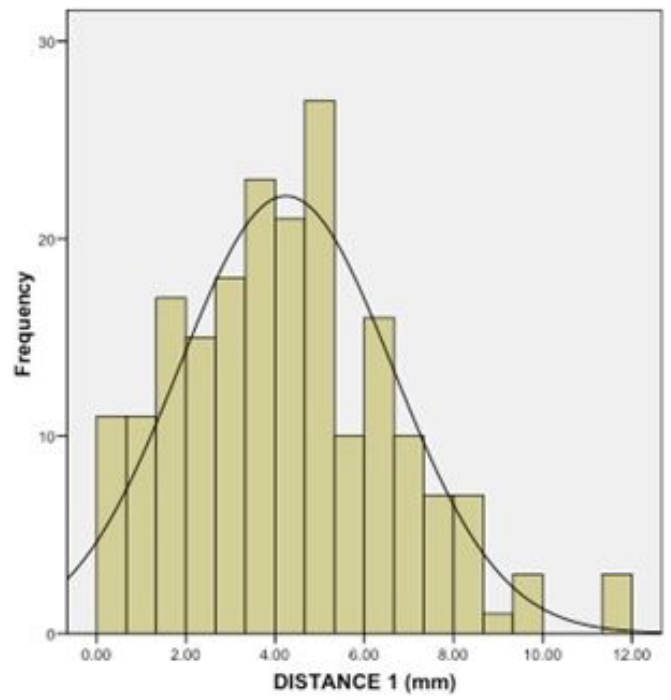

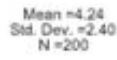

Figure 3. Distribution of the distances between the anterior maxillary sinus wall and the anterior border of the nasolacrimal fossa (distance 1).

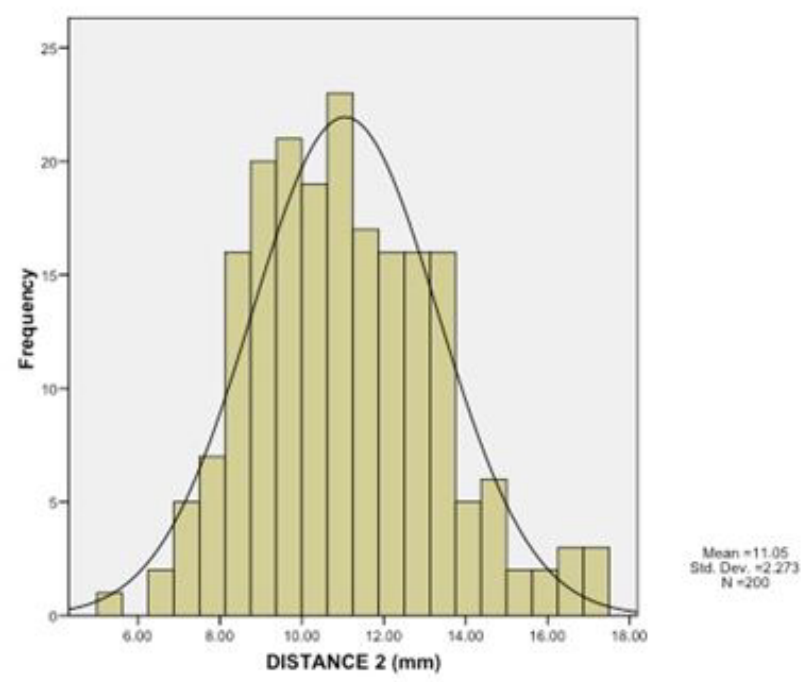

Figure 4. Distribution of the distances between the anterior maxillary sinus wall and the posterior border of the nasolacrimal fossa (distance 2).

\section{Results}

For the entire series the mean distance from the anterior wall of the maxillary sinus to the anterior border of the lacrimal fossa (distance 1) was $4.24 \pm 2.40 \mathrm{~mm}$ (minimum $0 \mathrm{~mm}$, maximum $11,62 \mathrm{~mm}$ ) (Figure 3). On the right and left side distances were $4.27 \pm 2.49 \mathrm{~mm}$ (minimum $0 \mathrm{~mm}$, maximum 11,62 $\mathrm{mm}$ ) and 4.21 $\pm 2.31 \mathrm{~mm}$ respectively (minimum $0 \mathrm{~mm}$, maximum $11.41 \mathrm{~mm}$ ) without statistical difference between the two sides ( $p$ 0.758). The mean distance from the anterior wall of the maxillary sinus to the posterior border of the lacrimal fossa (distance 2) was $11.05 \pm 2.27$ (minimum $5.45 \mathrm{~mm}$, maximum $17.32 \mathrm{~mm}$ ) (Figure 4), in which $11.07 \pm 2.35 \mathrm{~mm}$ (minimum $5.45 \mathrm{~mm}$, maximum $17.32 \mathrm{~mm}$ ) on the right side and $11.03 \pm 2.19 \mathrm{~mm}$ (minimum 6.31 $\mathrm{mm}$, maximum $17.32 \mathrm{~mm}$ ) on the left side. Again, there was no significant difference between the two sides ( $p>0.05 ; 0.948)$. The difference between distance 1 and distance 2 corresponds to the width of the lacrimal fossa. This width has a range between $4.00 \mathrm{~mm}$ and $10.88 \mathrm{~mm}$ with an arithmetic mean of $6.81 \mathrm{~mm} \pm 1.30 \mathrm{~mm}$.

To assess the proportion of patients in which a prelacrimal approach is readily feasible, distance 1 was subdivided into types I-III: Type I (0 -3 mm) was present in 63 (31.5\%) sites (Figure 5a), type II (>3mm $-7 \mathrm{~mm})$ in 112 (56\%) (Figure $5 \mathrm{~b}$ ) and type III (distance of $>7 \mathrm{~mm}$ ) in 25 (12.5\%) (Figure $5 \mathrm{c}$ ).

\section{Discussion}

Anatomy of the maxillary sinus reveals distinct hidden areas and niches that pose problems to endoscopic and instrumental access. Commonly it is a challenge to visualize and instrument every wall of the sinus even by highly experienced surgeons ${ }^{(7)}$. A study from Robey et al. ${ }^{(1)}$ came to the conclusion that the anterior wall and the floor (alveolar recess) are difficult to reach regardless of antrostomy or instrument selection.

An additional inferior antrostomy offers minor benefits with respect to control of the prelacrimal recess. In transnasal endoscopic skull base surgery it is also difficult to reach areas lateral in the pterygoid fossa and infratemporal fossa.

The areas of the maxillary sinus that are difficult to reach have been approached via the canine fossa, midfacial degloving approach or via a standard medial maxillectomy. However, these procedures have a higher morbidity rate compared to transnasal endoscopic approaches with a $75 \%$ transient and a $28 \%$ persistent rate of complications (pain, anesthesia, paresthesia, facial and dental numbness) ${ }^{(4,5)}$. As a result a modification of the endoscopic medial maxillectomy approach has become more and more popular by preserving the lacrimal apparatus and the inferior turbinate. The PLWA to the maxillary sinus allows optimal visualization of the most difficult areas within the maxillary sinus to be instrumented with $0^{\circ}$ degree endoscope and straight instruments and a very low morbidity overall ${ }^{(8)}$. Also in transnasal endoscopic surgery the lateral aspect of the pterygoid fossa and infratemporal fossa can be accessed using this approach ${ }^{(9)}$. To evaluate whether a PLWA can be performed, the preoperative $\mathrm{CT}$ scan has to be examined carefully because the distance of the lacrimal system to the anterior maxillary wall is critical when this approach is planned. If the window between the anterior wall of the maxillary sinus and the anterior border of the nasolacrimal duct is too small, a PLWA to the maxillary sinus is 


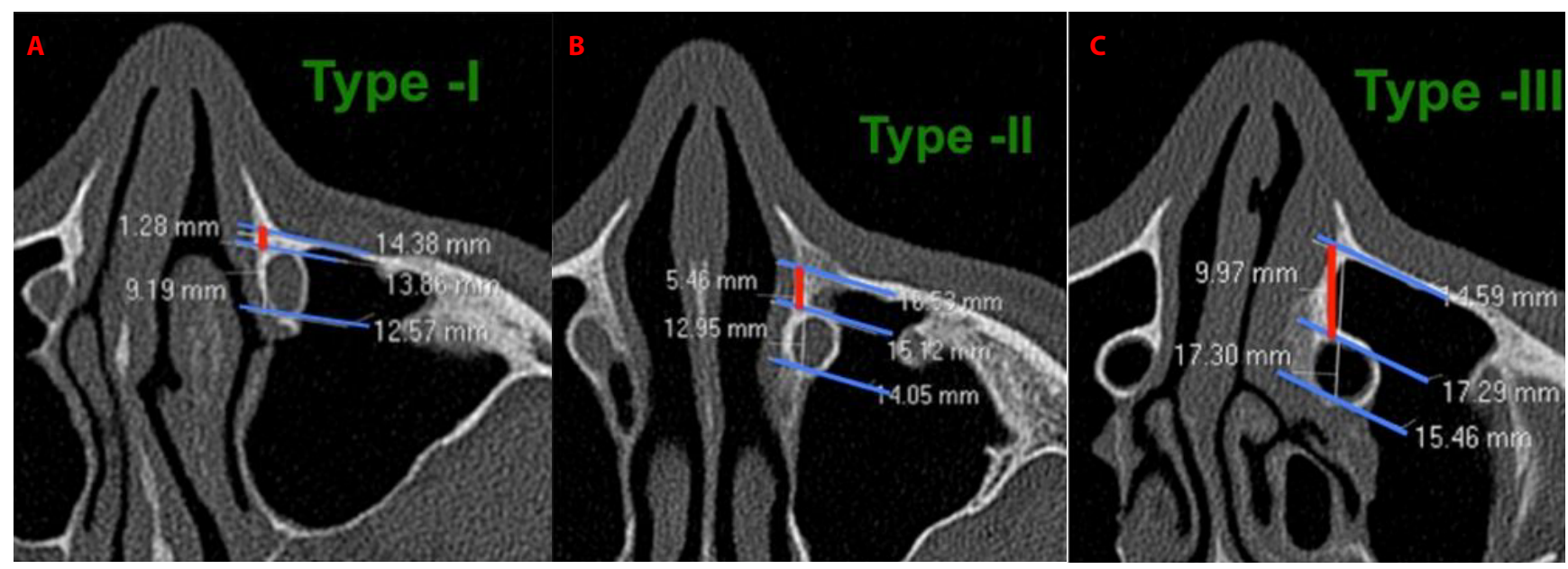

Figure 5. A) Type I: Distance 1 (anterior wall of maxillary sinus - anterior border of nasolacrimal duct) 0-3mm. B) Type II: Distance 1 (anterior wall of maxillary sinus - anterior border of nasolacrimal duct) >3-7 mm. C). Type III: Distance 1 (anterior wall of maxillary sinus - anterior border of nasolacrimal duct) $>7 \mathrm{~mm}$.

not possible without a temporary dislocation or even resection of the nasolacrimal duct. In these situations, much more bone needs to be removed and there more morbidity is likely. In order to evaluate in which patients a prelacrimal approach is possible without dislocation or resection of the nasolacrimal duct we divided the distance between the anterior wall of the maxillary sinus and the anterior border of the lacrimal fossa (distance 1) into three types which reflect the surgical complexity of a PLWA. In Type I (distance between 0 and $3 \mathrm{~mm}, 31.5 \%$ ), a prelacrimal approach is only possible with tear sac dislocation and a significant amount of bone removal. The window made by removal of bone will be small and it only allows limited access to the anterior wall of the maxillary sinus. In Type II (distance of $>3 \mathrm{~mm}-7 \mathrm{~mm}, 56 \%$ ), a prelacrimal approach is possible but only with bone removal along with dislocation of the tear sac. The window created can easily be closed with a soft tissue flap. In Type III (distance of $>7 \mathrm{~mm}, 12.5 \%$ ) a prelacrimal approach is readily performed with little bone work and an overview into the anterior wall and floor of the maxillary sinus is possible with little effort. In these situations, an approach to the lateral pterygoid and infratemporal fossa can also be created allowing direct visualization of these.

In a similar study looking at the relationship of the nasolacrimal duct and the anterior wall of the maxillary sinus, Navarro et al. ${ }^{(10,11)}$ evaluated the presence of a lacrimal recess and found a frequency of $30.9 \%-42 \%$. However, these studies focused on the presence of a prelacrimal recess and did not measure the distance from the anterior wall to the nasolacrimal duct. Overall, these findings are consistent with the finding of this study, which indicates, that the proportion of maxillary sinuses in which a PLWA is easy feasible is low and in the majority, a PLWA needs significant surgical effort.

\section{Conclusion}

The distance of more than $7 \mathrm{~mm}$ between the anterior border of the lacrimal duct and the anterior maxillary wall enables a straight forward prelacrimal window access to be done, but this is only possible in $12.5 \%$ of maxillary sinuses. Limited access (>3$7 \mathrm{~mm}$ ) is possible in $56 \%$ but often with tear sac disclocation. In $31 \%$ of maxillary sinuses $(0-3 \mathrm{~mm})$ prelacrimal window access requires temporary tear sac dislocation and a significant amount of bone removal.

\section{Authorship contribution}

DS, NV, HRB and BS developed the idea, contributed to the design of the study and wrote the paper. NV and BS collected the data and NV performed statistical analysis. NJ undertook a senior review of the paper.

\section{Conflict of interest}

The manuscript has not been published and is not being considered for publication elsewhere, in whole or in part or in any language. None of the authors have financial or other kinds of interests that might pose a conflict of interest in connection with the submitted article.

\section{References}

1. Robey A, O Brien EK, Leopold DA. Assessing current technical limitations in the small- hole endoscopic approach to the maxillary sinus. Am J Rhinol Allergy 2010; 24: 396-401. 2. Simmen. D, Jones N. Manual of endoscopic sinus and skull base surgery. NewYork: 2nd Edition. Thieme Medical Publishers; 2014. 3. Hosemann W, Scotti O, Bentzien S. 
Evaluation of telescopes and forceps for endoscopic transnasal surgery on the maxillary sinus. Am J Rhinol. 2003; 17: 311-6.

4. Vrabec DP. The inverted schneiderian papilIoma: a 25-year study. Laryngoscope. 1994; 104: 582-605

5. Maxfield AZ, Chen TT, Scopel TF, et al. Transnasal Endoscopic Medial Maxillary Sinus Wall Transposition with Preservation of Structures. Laryngoscope $2016 ; 126$ 1504-9.

6. Zhou B, Han D-M, Cui S-J, Huang Q, Wang C-S. Intranasal endoscopic prelacrimal recess approach to maxillary sinus. Chin Med J 2013; 126: 1276-1280.

7. Morrissey DK, Wormald PJ, Psaltis AJ. Prelacrimal approach to the maxillary sinus. Int Forum Allergy Rhinol 2016; 6: 214-218.
8. Suzuki M, Nakamura Y, Nakayama M, et al. Modified transnasal endoscopic medial maxillectomy with medial shift of preserved inferior turbinate and nasolacrimal duct. Laryngoscope 2011; 121:2399-2401.

9. Zhou B, Huang Q, Shen PH, et al. The intranasal endoscopic removal of schwannoma of the pterygopalatine and infratemporal fossae via the prelacrimal recess approach. J Neurosurg 2016; 124: 1068-73.

10. Navarro PL, Machado AJ, Crespo AN. Assessment of the lacrimal recess of the maxillary sinus on computed tomography scans. Eur J Radiol 2013; 82: 802-805.

11. Navarro L, Machado AJ, Crespo AN. Evaluation of the lacrimal recess of the maxillary sinus: an anatomical study. Braz $J$ Otorhinolaryngol 2013; 79: 35-38.
Daniel Simmen

Center for Otorhinolaryngology

Head and Neck Surgery

Klinik Hirslanden

Witellikerstrasse 40

CH-8032 Zurich

Switzerland

Tel: ++41443872800

Fax: ++41443872802

E-mail:simmen@orl-zentrum.com 